\title{
Monitoring and modeling the deposition of metal nanoparticles on surfaces by impedance
}

DOI:

10.1016/j.apsusc.2020.148806

\section{Document Version}

Accepted author manuscript

Link to publication record in Manchester Research Explorer

\section{Citation for published version (APA):}

C. Hensel, R., H. Gonçalves, M., L. Rodrigues, K., Abrao Oiko, V. T., do L. Pimentel, V., A. Pereira-da-Silva, M., Hillenkamp, M., Riul Jr., A., \& Rodrigues, V. (2020). Monitoring and modeling the deposition of metal nanoparticles on surfaces by impedance. Applied Surface Science, 544(148806). https://doi.org/10.1016/j.apsusc.2020.148806

\section{Published in:}

Applied Surface Science

\section{Citing this paper}

Please note that where the full-text provided on Manchester Research Explorer is the Author Accepted Manuscript or Proof version this may differ from the final Published version. If citing, it is advised that you check and use the publisher's definitive version.

\section{General rights}

Copyright and moral rights for the publications made accessible in the Research Explorer are retained by the authors and/or other copyright owners and it is a condition of accessing publications that users recognise and abide by the legal requirements associated with these rights.

\section{Takedown policy}

If you believe that this document breaches copyright please refer to the University of Manchester's Takedown Procedures [http://man.ac.uk/04Y6Bo] or contact uml.scholarlycommunications@manchester.ac.uk providing relevant details, so we can investigate your claim.

\section{OPEN ACCESS}




\title{
Monitoring and modeling the deposition of metal nanoparticles on surfaces by capacitance
}

\author{
Rafael C. Hensel ${ }^{\mathrm{a}}$, Maria H. Gonçalves ${ }^{\mathrm{a}}$, Kevin L. Rodrigues ${ }^{\mathrm{a}, \mathrm{b}}$, Vitor T. A. \\ Oiko $^{\text {a,c }}$, Vinicius do L. Pimentel ${ }^{\mathrm{d}, \mathrm{e}}$, Marcelo A. Pereira-da-Silva, ${ }^{\mathrm{f}, \mathrm{g}}$, Matthias \\ Hillenkamp $^{\mathrm{a}, \mathrm{h}}$, Antonio Riul Jr. ${ }^{\mathrm{a}}$, Varlei Rodrigues ${ }^{\mathrm{a}, *}$ \\ ${ }^{a}$ Instituto de Física "Gleb Wataghin", Universidade Estadual de Campinas, 777 Sérgio \\ Buarque de Holanda Street - Cidade Universitária Zeferino Vaz, Campinas - SP, Brazil. \\ ${ }^{b}$ Departamento de Física Instituto de Ciências Exatas, Universidade Federal de Minas \\ Gerais, 6627 Av. Pres. Antônio Carlos, Belo Horizonte - MG, Brazil. \\ ${ }^{c}$ The University of Manchester, Oxford Road, Manchester, M13 9PL, United Kingdom. \\ ${ }^{d}$ Centro de Tecnologia da Informação Renato Archer, Rodovia Dom Pedro I (SP-65), Km \\ 143,6, Campinas - SP, Brazil. \\ ${ }^{e}$ Faculdade de Engenharia Elétrica e de Computação, Universidade Estadual de Campinas, \\ 400 Albert Einstein Avenue - Cidade Universitária Zeferino Vaz, Campinas - SP, Brazil. \\ ${ }^{f}$ Instituto de Física de Sao Carlos - IFSC/USP, São Carlos - SP, Brazil. \\ ${ }^{g}$ Centro Universitário Central Paulista - UNICEP, São Carlos - SP, Brazil. \\ ${ }^{h}$ Institute of Light and Matter, University Lyon, Université Claude Bernard Lyon 1, CNRS, \\ UMR5306, F-69622 Villeurbanne, France.
}

\begin{abstract}
The study of metallic nanoparticles (NPs) on surfaces and their properties has become a common subject for a variety of areas. Notably, in order to exploit the unique intrinsic features of deposited NPs, samples having low coverage are required. The sample characterization by electrical techniques has, however, so far been limited mostly to systems near or beyond the percolation limit, when the film's resistive behavior is dominant. Here we describe the capacitative response of interdigitated electrodes (IDE) during Ag NP deposition, from the very beginning up to the percolation limit. Our experiments present two regimes: up to $20 \%$ of coverage the capacitance grows linearly with the deposition, increasing abruptly afterward. To understand the experimental data, we propose a model in which the linear response is attributed to isolated and agglomerated NPs. Initially, isolated NPs contribute to the capacitive response. Beyond $\sim 20 \%$
\end{abstract}

\footnotetext{
* Corresponding author

Email address: varlei@ifi.unicamp.br (Varlei Rodrigues)
} 
coverage, shielded regions of the IDE due to agglomerated NP islands start to dominate. These interpretations are supported by Electron Microscopy and Atomic Force Microscopy. The proposed analysis allows improving the control of the concentration of NP deposited on surfaces systems with low coverage by monitoring the capacitance.

Keywords: magnetron sputter nanoparticle deposition, mass selected nanoparticle, nanoparticle thin fims, in-situ capacitance measurement, silver, capacitance behavior modeling

\section{Introduction}

Nanostructuring paved the way to a plethora of exciting possibilities, from basic studies to new applications. These systems can present properties that differ sharply from their macroscopic scale counterpart or even show new and

5 unexpected effects. Metallic nanoparticles (NP) and thin films, both examples of systems widely studied in this context, show optical [1, 2, 3], electrical [4] and catalytic [5] properties with great potential for new technological applications. Significant advances have been achieved in studying the electrical properties of metallic NP films. 6, 7] Most of these studies are based on samples deposited by atom deposition from the sputtering of metal targets. [8, 9 However, in this process, the nanostructure growth depends on the diffusion of metal atoms on the surface of the substrate. 10] In this context, the control, and modeling of the electrical properties of metallic NP thin films is crucial for the development and miniaturization of electronic devices and flexible electronics.

Electrical properties of metal films on surfaces obtained by the deposition of atoms are very complex because of the complicated interplay of different parameters like atom and island mobility on the surface, wettability and others [11, 12, 13, 8, 9]. Furthermore, films with metallic NPs as building blocks also present nanoscale and disorder effects that modify the electrical properties

20 14, 15. J. E. Barborini et al. deposited Fe, Pd, Nb, W, and Mo clusters on interdigitated electrodes (IDEs), planar devices composed of metallic digits on 
an insulating substrate, and performed DC electrical measurements during the film formation. They correlate the device resistivity evolution with the conduction path according to the formed film's mean thickness. They show that the resistivity decreases two orders of magnitude, due to the transition from 1D to $2 \mathrm{D}$ conductive path. The resistivity reaches a minimum, and then increases due to the formation of a 3D conductive path [14. A. Sattar et al. describe how quantum conductance can lead to switching behavior in percolating nanoparticle films [15]. O. Kylián et al. report that the resistivity of Pt nanoclusters film on Si and glass decreases three orders of magnitude while the film thickness increases up to $50 \mathrm{~nm}$, reaching $3 \times 10^{-3} \Omega \mathrm{cm}$ [10. Recently, there were several reports on cluster assembled nanostructures that present memristive behavior. C. Minnai et al. reported switching behavior in Au cluster-assembled nanostructured films on glass substrates, which is attributed to the formation/destruction 35 of conductive paths between particles [16. M. Mirigliano et al. have reported the resistance evolution as a function of Au NP film thickness beyond the percolation limit and applied percolation theory to evaluate how the variation of nanoagregates connectivity influence the memristive effect [17. Recently, A. Vahl et al. applied conducting atomic force microscopy to investigate in situ individual NPs alloys memristive effect and their structural degradation [18.

The vast majority of studies on electrical properties of NP films are based on DC measurements [14, 15, 19, 20, 21], that present no conductivity between low surface coverage and the percolation limit. However, many studies on metallic NPs deposited on a surface, exploring magnetic [22, 23], optical [3], and catalytic

45 24] properties, are carried out in a low concentration regime, where the interactions between NPs are negligible in order to preserve the intrinsic nanoscale properties of the NPs [25, 26, 27]. In this article, we report the experimental methods for in situ monitoring of Ag NP's deposition between the fingers of the IDEs via capacitance measurements. This technique allows the IDE electrical characterization from the very start of deposition up to the percolation threshold. To understand the experimental data, we propose a model based on how randomly distributed metallic NPs affect the electric field between IDE digits, 
which can also be translated as variations in the capacitance. The methodology proposed here allows for better control and interpretation of metallic NP deposited on surfaces. These approaches can be further applied on other surfaces, such polymers. The incorporation of metallic NP into a polymer system has been exploited to tune sensor sensitivity and selectivity [28, 29, 30, 31] and suppress lithium dendrite growth in lithium metal batteries [32], for example. Nevertheless, so far, NP deposition on polymer has been achieved, leaving crucial parameters, like concentration, out of reach. In this sense, the NP deposition's electrical monitoring is essential to define NP concentration, which usually stays far below the percolation limit to better define the useful feature of these devices.

\section{Materials and Methods}

\subsection{In situ capacitance monitoring of IDE during NP deposition}

We fabricated NP using a gas aggregation source 33. In this system, the erosion of a metal wire target produces a cloud of atoms. Collisions between sputtered atoms and cold Ar atoms promote NP formation. Figure 1(a) shows a scheme of the experimental setup used for the NP fabrication. A set of electrostatic lenses guides and focuses the positively charged NP beam onto the sample holder [34]. The set of lenses includes a Bessel box, allowing the selection of the NP mean size to be deposited on the sample holder. A fraction of the NPs produced are deflected to a time-of-flight mass spectrometer (TOF) that keeps track of the NP size distribution during the whole experiment. The major fraction of the NPs is deposited on the sample holder or on a current detector with

75 a $200 \mathrm{~mm}^{2}$ area that monitors the charged NP deposition rate. To estimate the area covered by NPs, we consider the spherical NP projection in circles of area $A_{N P}$. We estimate the NP covered area according to $\frac{A_{N P}}{A_{S E N S O R}} \frac{t \times I}{e}$, in which $\frac{A_{N P}}{A_{S E N S O R}}$ is the ratio between the NPs area and the current sensor area, $t$ is the deposition time, $e$ is the electron's charge and $I$ is the NPs deposition current.

80 The produced NP deposition current was monitored using a current detector connected to Keithley Series 6487 Picoammeters, which presented a mean value 
of $I=330 \mathrm{pA}$. Besides, the NP mean diameter was monitored during the whole experiment using the TOF, which presented a mean diameter of $\sim 3 \mathrm{~nm}$.

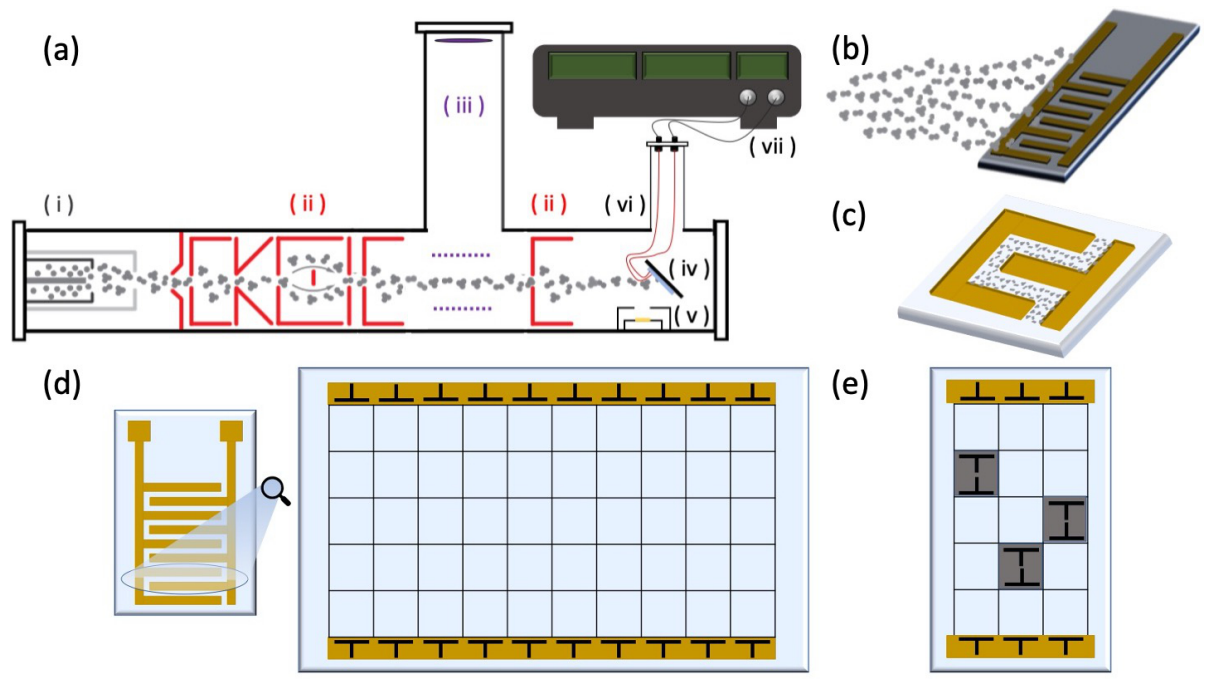

Figure 1: (a) Experimental setup scheme of the atomic cluster source connected to a capacitance meter system. (i) Aggregation chamber where atoms are sputtered from a wire target and start aggregating in a low-temperature Ar atmosphere; (ii) electrostatic optics that guide, mass select and focus the NPs; (iii) time-of-flight mass spectrometer (TOF) for in-situ analysis of produced NPs size; (iv) $45^{\circ}$ sample holder where IDE is mounted for in-situ capacitance monitoring; (v) neutralization filament to avoid charging effects on the sample; (vi) deposition chamber; (vii) capacitance meter setup. (b) Sample holder details to illustrate the NPs deposition on the IDE. Note that the IDE is rotated with respect to the sample holder position to better represent the deposition. (c) Although the NPs are deposited everwhere on the IDE, for clarity we show only the NPs deposited between adjacent digits. Sketch to illustrate the region between adjacent digits of the IDE to show (d) the parallel association of capacitors, (e) and the series capacitor after the sites (grey) being filled with nanoparticles.

To investigate the Ag NP film electrical properties, we have deposited the produced NPs onto IDEs. Here, we used gold IDEs on glass, comprising 30 pairs of digits with $3 \mathrm{~mm}$ length, $200 \mathrm{~nm}$ height, $40 \mu \mathrm{m}$ width, and spaced 40 $\mu \mathrm{m}$ each other, fabricated at the Microfabrication Laboratory, National Center for Research in Energy and Materials, Brazil. We performed capacitance measurements to analyze in situ the evolution of the electrical properties of IDEs 

situ avoids NP oxidation, which is an important issue when using such small NPs [35. The produced NPs are randomly deposited on the whole IDE, and the NPs deposited on the IDE digits do not modify the overall electrical properties. However, the NPs deposited between adjacent digits, equivalent to half of the total IDE area, do modify the electrical response, as illustrated in figure 1(c) We perform capacitance measurements using a homemade setup, described in detail elsewhere 36 . Briefly, a sinusoidal voltage of $1 \mathrm{~V}$ amplitude at $1 \mathrm{kHz}$ is applied to one comb of digits of the IDE [36]. The current from the pick-up electrode is amplified using an operational amplifier in a differential configuration. A lock-in system measures the output voltage and the phase shift between input and output signals in $0.5 \mathrm{~s}$ intervals. We interpret the measurements using an equivalent circuit composed of a parallel association of capacitive and resistive components 36] The metal NP deposition between the IDEs digits modifies the device geometry. For instance, considering that the region between adjacent digits can be approximated to a parallel association of capacitors, the nanoparticle deposition decreases the gap of the capacitors, which is equivalent to having an series association of capacitors, as shown in Figure 1 (d), and (e), respectively. Consequently, increasing the total capacitance.

\subsection{Ex situ morphology investigation}

We investigated the coverage and the topography of the NP film deposited on the IDE to interpret the electrical behavior observed. After the NP deposition, we analyzed the topography of the region between the IDE's digits using Atomic Force Microscopy (AFM), in a BRUKER Dimension ICON instrument, using a rectangular shaped silicon tip, $42 \mathrm{Nm}^{-1}$ spring constant and $330 \mathrm{kHz}$ free oscillation in tapping mode. Complementary information about the NP size evolution is obtained from Transmission Electron Microscopy (TEM) experiments. The NP size distribution and covered area on a surface were investigated on samples produced by the deposition of Ag NP for different deposition times on Ultrathin Carbon Film Supported by a Lacey Carbon Film on 400 Mesh Copper TEM 
grids. These grids were analyzed using a Jeol 2100F field emission gun TEM at Electron Microscopy Laboratory, National Center for Research in Energy and Materials, Brazil.

\section{Results}

\subsection{Capacitance monitoring of silver nanoparticle deposition}

The capacitance evolution, as a function of the fraction of area covered by the NPs, from the very beginning up to $50 \%$ coverage, is shown in figure 2. At the beginning of the deposition, the measured capacitance value was $C_{\mathrm{IDE}}=10.25 \mathrm{pF}$, corresponding to the bare IDE in vacuum [37, 38]. A monotonic increase of capacitance following the NP coverage is observed even during the early stages of the deposition, demonstrating higher sensitivity than what is usually seen in DC measurements of resistivity [14, 9]. Besides, figure 2 allows us to infer that the capacitance measurements presents two growth regimes: an initial linear growth for the first $20 \%$ of the covered area, which becomes superlinear after $20 \%$ of covered area. The measurement was stopped after one hour of deposition, which was equivalent to $50 \%$ of the estimated covered area.

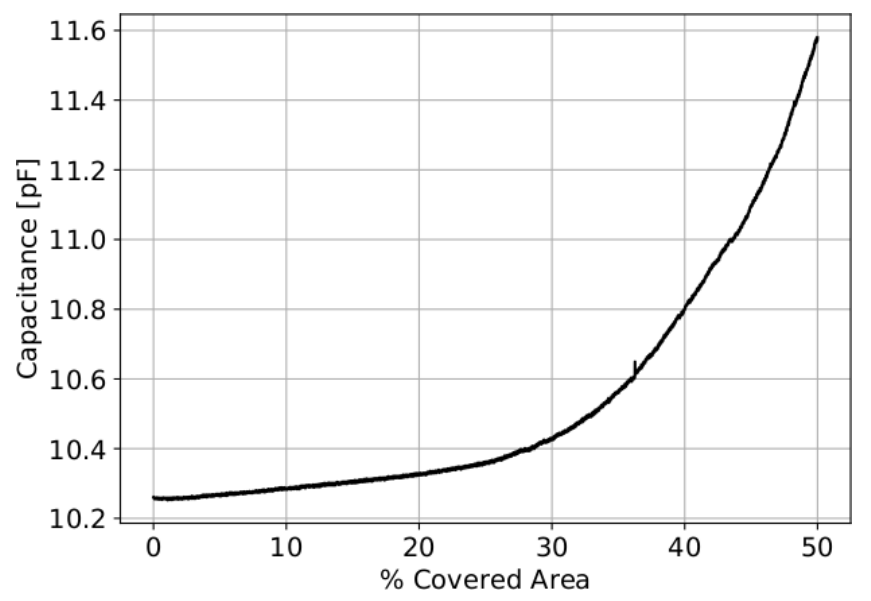

Figure 2: IDE capacitance variation as a function of the covered area during the deposition of $3 \mathrm{~nm}$ silver NPs. 

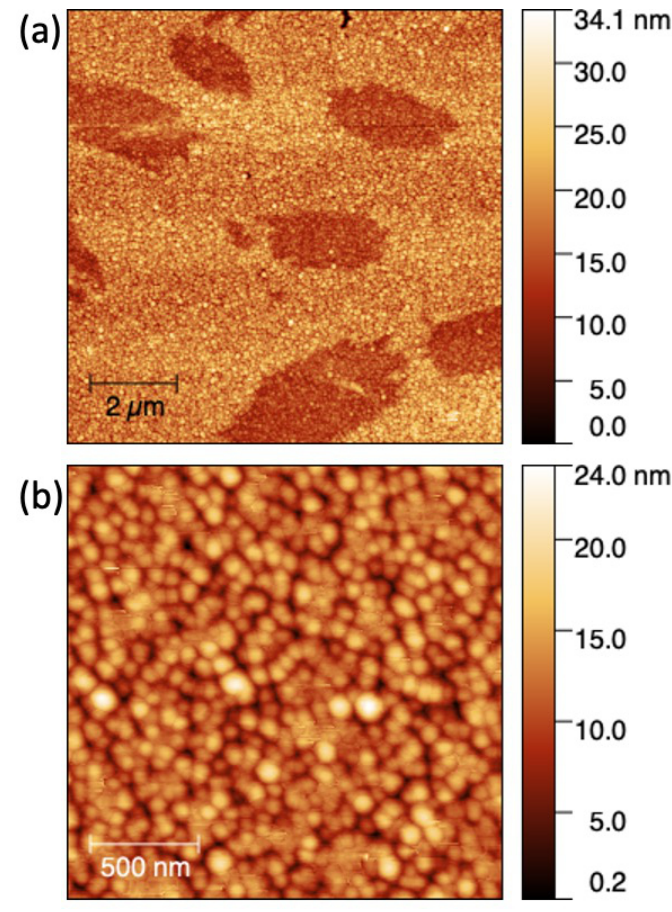

Figure 3: (a) $10 \mu \mathrm{m} \times 10 \mu \mathrm{m}$ and (b) $2 \mu \mathrm{m} \times 2 \mu \mathrm{m}$ AFM micrograph between IDE digits after the NP deposition. Note that the holes are related to incompletely filled NP layers.

\subsection{Morphological investigation of silver nanoparticle on surfaces}

To analyze the topography between adjacent digits, we performed an AFM analysis. Figure 3 shows an AFM micrograph of the IDE used to obtain the capacitance behavior shown in figure 2. As shown in figure 3(a), the deposited NPs form an almost continuous thin film, with some incompletely filled islands. The mean height measurement in the depression regions is $(4.0 \pm 0.5) \mathrm{nm}$, which is equivalent to the NP diameter. Figure 3(b) was obtained where the NPs form a seemingly continuous film. We can observe that the NPs do not form an ordered packed film as assumed for the covered area estimation, but they are randomly distributed on the surface. The micrograph also shows that the NPs start to agglomerate on top of the unfilled layer of previously deposited NPs.

Figures 4 (a-c) present TEM micrographs of Ag NPs for 1.5\%, $5 \%$, and $11 \%$ covered area, respectively. We observe that an increasing percentage of the 
(a)
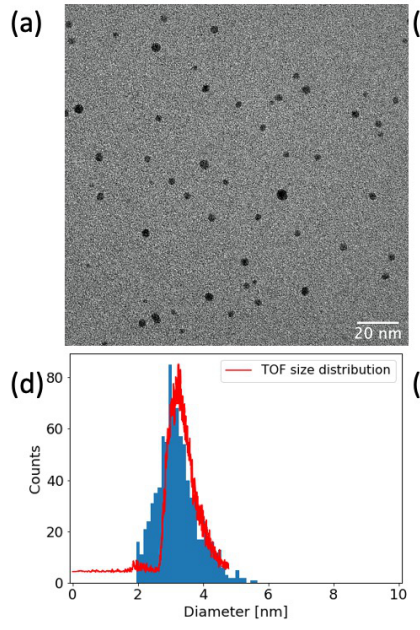

(b)
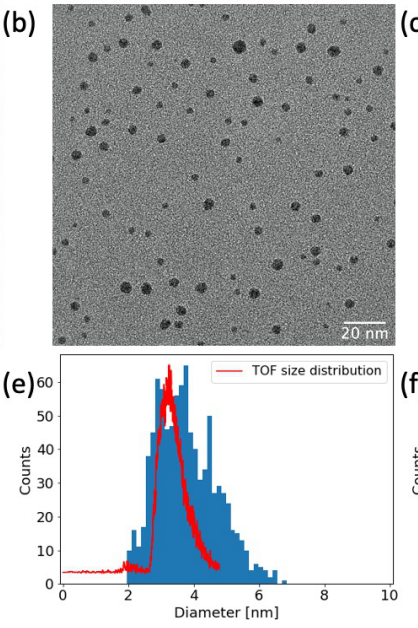

(c)
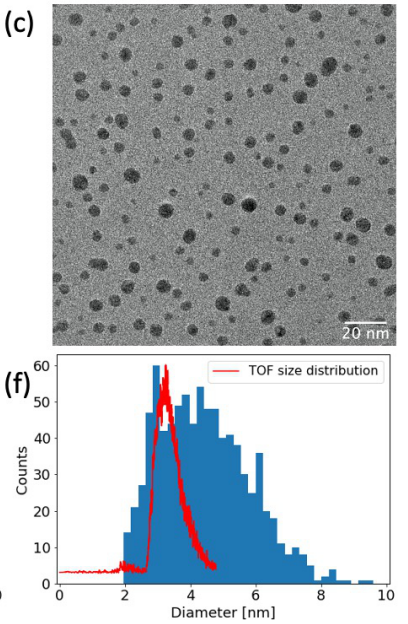

Figure 4: TEM micrographs of Ag NPs: (a) 1.5\% covered area, (b) 5\% covered area, (c) $11 \%$ covered area. We can observe that the NPs coalesced on the grid and increased their diameter with the covered area. Size distribution for (d) $1.5 \%$, (e) $5 \%$ and (f) $11 \%$ covered area. The red curve is the time-of-flight size distribution during the sample preparation, which presents $3.2 \mathrm{~nm}$ for the NPs mean diameter.

covered area also increases the NP diameter due to coalescence. The size distribution obtained for these micrographs are compared with the time-of-flight size distribution, shown in graphs $4(\mathrm{~d}-\mathrm{f})$. We can see that for $1.5 \%$ covered area, the NP size distribution derived from the TEM analysis coincides with the TOF size distribution obtained during deposition (figure 4 (d)). Increasing the percentage of covered area shifts the NPs mean size and broadens the distribution towards bigger NPs, as shown in figures $4(\mathrm{e})$ and (f).

\section{Model}

\subsection{Capacitance evolution during silver nanoparticle deposition}

The capacitance monitoring during the $3 \mathrm{~nm} \mathrm{Ag} \mathrm{NPs} \mathrm{deposition} \mathrm{on} \mathrm{a} \mathrm{bare}$ IDE shows a capacitance growth profile with the covered area, figure 2 In contrast to DC measurements, the capacitance measurements starts to increase from the very beginning of the deposition, which corresponds to the contribution of isolated NPs to the device capacitance. The variation in capacitance due to 
the NPs occurs because of the change in the electric field they cause when deposited in the area between digits. The IDE capacitance can be represented by an equivalent circuit of parallel capacitors that describe the contribution from different parts of the IDE to the total capacitance. For example, one capacitor can take into account the contribution from the top of the digits, and another the region between digits, where the field is parallel to the IDE substrate. Different parallel capacitors then describe this last region. In this sense, deposited NPs only affect one of these capacitors, representing a fraction of the capacitance due to the region between digits and close to the substrate surface. All other contributions remain constant during the experiment. In this sense we can write the total capacitance as a function of the covered area $A$ by $C_{\text {total }}(A)=C(A)+C_{0}^{\prime}$ where $C(A)$ was the capacitance due to the NP 175 deposition and $C_{0}^{\prime}$ the part that remained constant throughout the deposition.

To interpret the observed capacitance behavior, we propose a model based on a simulation of the evolution of the capacitance during the random filling of a rectangular mesh grid that represents the area between the IDE digits with NPs, as illustrated in figure 5, where grid positions are filled randomly to mimic NP deposition over time. The digit spacing $S$ and length $L$ is divided in squares of side $\Delta S=\frac{S}{n}$ and $\Delta L=\frac{L}{m}$, respectively. To keep the device aspect ratio of the region between digits, we use $m=75 n$. Each cell is addressed with a pair of indices, $(i, j)$, with $i \in[1, n]$ and $j \in[1, m]$ for the spacing $(S)$ and length $(L)$ positions, respectively. Each column $j$ is associated with a capacitor $C_{j}$ of parallel plates, as depicted in figure 5 . The gap between digits corresponds to the distance between the plates of each $C_{j}$ capacitor. The capacitance of this device is thus equivalent to an array of parallel capacitors $C_{j}$, i.e., $C(A)=\sum_{j=1}^{m} C_{j}(A)$. Therefore, $C(A=0 \%)=C_{0}$, is the initial value of this capacitance in vacuum, before any deposition. Let a particle be introduced at row $i$ and column $j$ in the grid. When $i$ is the first or last available space in column $j(i=1$ or $i=n)$, the capacitance $C_{j}$ changes as if the capacitor's gap was reduced by one grid space $\Delta S$. If instead the particle is introduced at an intermediate space $1<i<n$, the capacitance $C_{j}$ becomes the series association of two parallel capacitors with 
gaps $(i-1) \Delta S$ and $(n-i) \Delta S$. Note that the total sum of the gaps is also reduced by one grid space like before, but now it created two capacitors. In figure 5 red squares illustrate sites filled with NPs.

We must also take into account that NPs agglomerate and that they also touch the digits, as illustrated in figure 5 . In these situations, empty sites can be shielded from the electric field by sites occupied by NPs. The dark blue squares in figure 5 illustrate situations in which empty sites are shielded by agglomerations of NPs, with or without contact with the digits. In the model proposed for $C(A)$, groups of adjacent particles with at least one connection to a digit will shield all empty sites between any constituent particles and the nearest wall. Shielded sites contribute to $C(A)$ as if they were occupied.

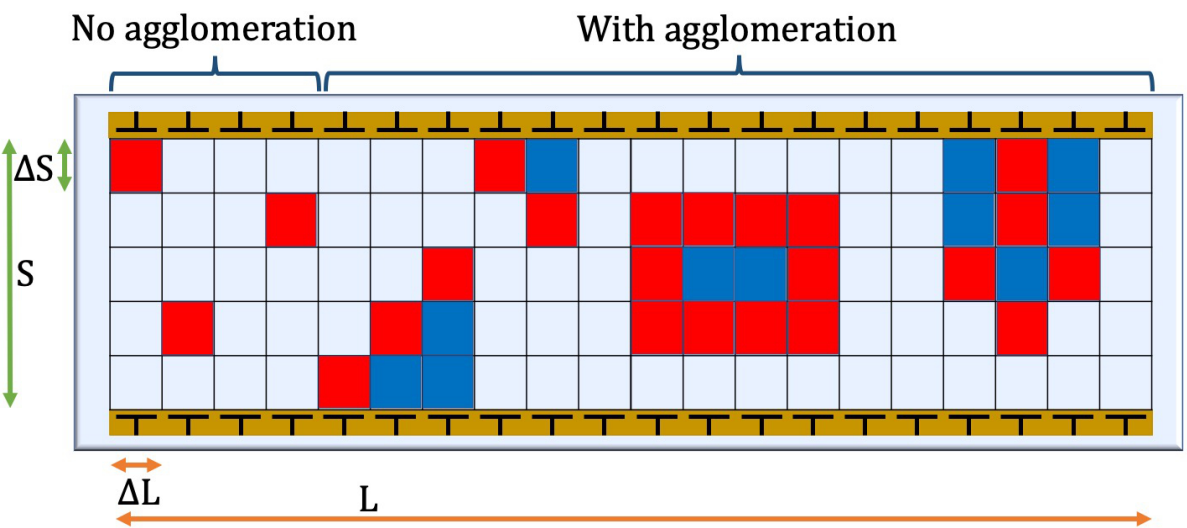

Figure 5: Schematic of the rectangular mesh depicting regions with (right-hand side) and without (left-hand side) agglomeration of NPs. Light blue squares are empty sites; red squares are filled with NPs and dark blue squares are the shielded positions. The simulations discussed in the text were performed for larger meshes $(S=\Delta S n$ with $\mathrm{n}=50)$.

In our experiment, it is not possible to separate $C(A)$ from the constant capacitance value, $C_{0}^{\prime}$. As $C(A)$ is proportional to the initial value $C(A=0 \%)=$ $C_{0}$, we can rewrite it as $C(A)=k(A) C_{0}$. So, we adjusted the proposed model for $C(A)$ to the experimental curve considering that $C_{\exp }(A)=k(A) C_{0}+C_{0}^{\prime}$ and find the values for $C_{0}$ and $C_{0}^{\prime}$ to evaluate the total capacitance as a function of $A$ using our proposed model. 


\section{Discussion}

In the previous section, we have proposed a model to calculate the evolution of the conductance as a function of the covered area. For the calculations, we used a mesh with $\mathrm{n}=50$. We also performed the calculations for a bigger mesh,

which increases the computation cost but yields compatible results. Figure 6(a) shows the experimental data, the capacitance simulated without considering the agglomeration of NPs, the simulated considering shielded regions due to agglomerated NPs, and the linearly adjusted region used to normalize both simulations to the experimental data. Figure 6(b) presents the number of agglomerations and effective coverage as a function of the covered area. The former is for the number of isolated NPs and the number of groups of NPs that are considered agglomerations in our models. After sorting a position in the matrix to be filled, our method analyzes the whole matrix and counts the number of agglomerations in each iteration. The number of agglomerations increases until reaching approximately $20 \%$ coverage. Beyond $20 \%$ coverage, new deposited NP falls on or connect pre-formed agglomerations, decreasing the number of agglomerations, leading to the bell-like dependence of the number of agglomerations as a function of the covered area seeing in figure 6(b). The effective coverage refers to the sum of sites in the grid containing an NP or shielded by an agglomeration.

We see in figure 6(a) that experimental and simulated results are in good agreement when the linear portion of the curve is considered (up to $20 \%$ of coverage). It is important to remark that the model without agglomeration is, in fact, a linear capacitance function of the covered area up to $50 \%$ coverage, and therefore, it is incapable of describing the non-linear behavior observed for coverages higher than $\sim 20 \%$. Moreover, figure 6(b) showed that the number of isolated and agglomerated NPs grows until $\sim 20 \%$ of coverage and then goes down. Also, the $\%$ effective coverage was linear until $\sim 20 \%$ of the covered area and started to increase rapidly. This behavior indicates that initially, the IDE's capacitance can be described by the effects of isolated NPs.

From $20 \%$ coverage onwards, the capacitance begins to be dominated by the 

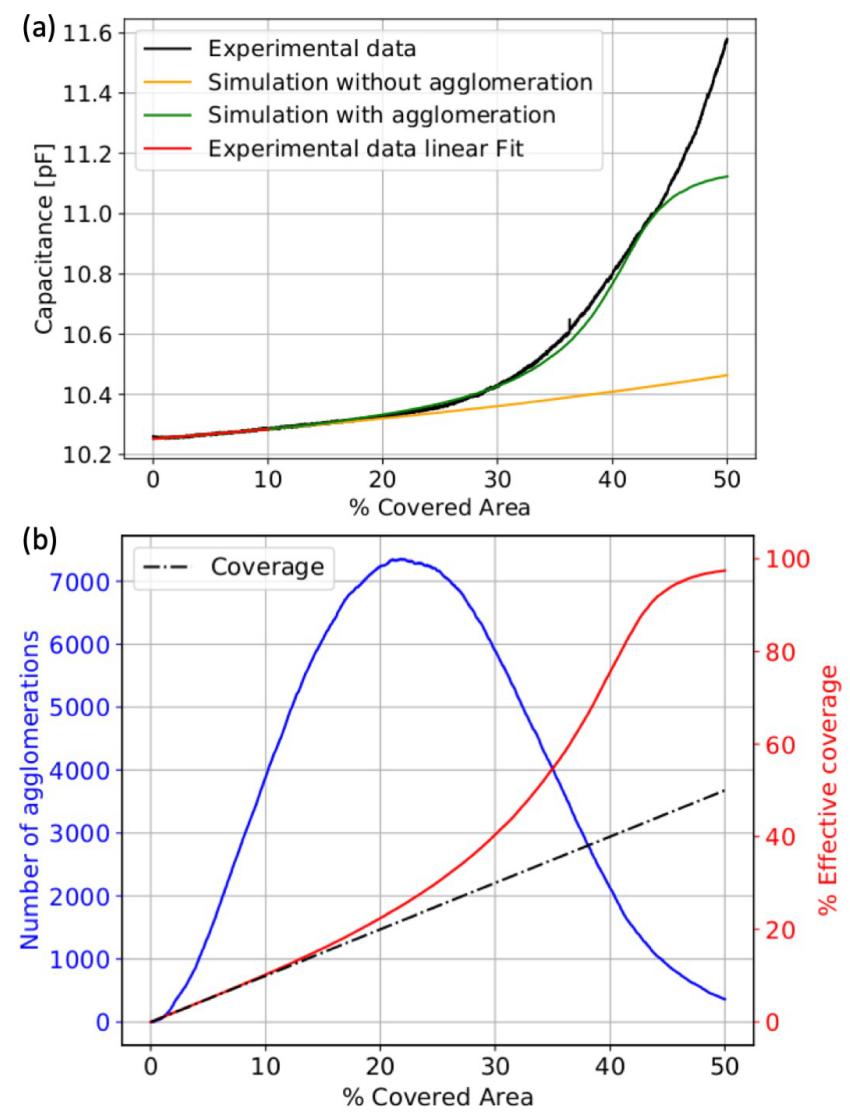

Figure 6: (a) Experimental and simulated values of IDE capacitance during NPs deposition. (b) Left: the sum of the number of isolated NPs and the number of agglomerated NPs; right: $\%$ of occupied and shielded positions on the grid. The dashed line describes the $\%$ covered area, also associated with right-hand axis.

existence of clusters of NPs that shield regions of the IDE. This can be verified in figure 6(a), where the simulation with shielded sites from agglomerated NPs (green curve) follows the experimental data up to $\sim 45 \%$ coverage. Besides, from $\sim 20 \%$ coverage, the number of agglomerations decreases, indicating that isolated NPs as well as agglomerations start to touch, being considered as coalescence. Also from this point on, the $\%$ of effective coverage is no longer linear with the $\%$ of the covered area, but grows faster. This result indicates that the contribution of shielded areas, due to NP agglomeration, must be considered in 
order to understand the IDE capacitance behavior from $20 \%$ coverage.

Above $45 \%$ coverage, the IDE capacitance in our model saturates, not correctly representing the experimental data. This can be related to the percolation threshold, which adds a resistive component to the device. According to the literature, the percolation occurs for $40 \%$ coverage in a square matrix with next-nearest neighbour conductance, from this point on our model is no longer adequate [39. Besides, the $\%$ of effective coverage also saturates (occupied and shielded positions on the grid). We recall the literature showing a measurable conductive component in electrical transport for over $45 \%$ coverage [40, which must contribute to the measurement of our IDE electrical behavior.

We furthermore note here that our simple model correctly reproduces not only the overall trend but can even quantitatively describe the variations in capacitance despite its simplifications. From the AFM image in Figure 3 we can deduce that three-dimensional growth is non-negligible; we have, however, tested that permitting a second layer of deposited NPs in the model has only minor effects. This is comprehensible as additional layers only slightly re-scale the abscissa of Fig. 6

\section{Conclusions}

Surface deposition of metal NPs for numerous studies, on the one hand, relies on controlling the low concentration of NP on the substrate. On the other hand, works on the electrical properties of films of NPs usually investigate films that are close to or above the percolation limit, where electric transport is predominately resistive. We have focused in our study on the evolution of the sample capacitance from the very beginning of the NP deposition up to the percolation limit. The observed variation in capacitance can be divided into two regimes, a linear one between $0 \%$ and $\sim 20 \%$ coverage, and a non-linear one for values above $\sim 20 \%$. To interpret these results, we propose a simple model based on the occupation of sites between digits. The model takes into account how agglomeration of NPs leads to the formation of shielded sites that affect the 
electric field between electrodes and the capacitance consequently. This model shows that the first capacitance regime is dominated by isolated and agglomerated NPs. Above $\sim 20 \%$ coverage, the capacitance starts having contributions of shielded regions between the electrodes due to agglomerated NP islands, leading to a super-linear capacitance increase. TEM and AFM micrographs support our interpretations. The proposed model and electrical behavior analysis open possibilities to control the concentration of NP deposited on surfaces for optical, magnetic, and catalytic studies. Besides, our work contributes to the development and miniaturization of electronic devices and flexible electronics based on IDEs with NPs film. It can also be extended to the deposition of wet-chemically synthesized NPs, facilitating the comprehension of several phenomena and effects caused by NP introduction in a variety of applications in several knowledge areas.

\section{Acknowledgements}

The authors are grateful to FAPESP (FAPESP, 2014/03691-7, 2013/14262-7, 2016/12807-4), CNPq, and CAPES for financial support. Experimental support from the Microfabrication Laboratory (proposal LMF-18748 and LMF-21855) and Electron Microscopy Laboratory (proposal TEM-21702, ME-22308, TEM23432, TEM-24315, TEM-C1-25093) at Brazilian Nanotechnology National Laboratory (LNNano), CNPEM, Campinas, Brazil for their support with the use of equipaments.

\section{References}

[1] R. A. Maniyara, D. Rodrigo, R. Yu, J. Canet-Ferrer, D. S. Ghosh, R. Yongsunthon, D. E. Baker, A. Rezikyan, F. J. García de Abajo, V. Pruneri, Tunable plasmons in ultrathin metal films, Nature Photonics 13 (5) (2019)

328-333. doi:10.1038/s41566-019-0366-x.

URL https://doi .org/10.1038/s41566-019-0366-x 
305 [2] G. Ding, C. Clavero, D. Schweigert, M. Le, Thickness and microstructure effects in the optical and electrical properties of silver thin films, AIP Advances 5 (11) (2015) 117234. doi:10.1063/1.4936637.

URL https://doi.org/10.1063/1.4936637

[3] A. Campos, N. Troc, E. Cottancin, M. Pellarin, H.-C. Weissker, J. Lermé, M. Kociak, M. Hillenkamp, Plasmonic quantum size effects in silver nanoparticles are dominated by interfaces and local environments, Nat. Phys. 15 (2019) 275-280. doi:10.1038/s41567-018-0345-z. URL https://doi.org/10.1038/s41567-018-0345-z

[ [4] H. Wei, H. Eilers, From silver nanoparticles to thin films: Evolution of microstructure and electrical conduction on glass substrates, Journal of Physics and Chemistry of Solids 70 (2) (2009) 459-465. doi:https://doi.org/10.1016/j.jpcs.2008.11.012.

a URL http://www.sciencedirect.com/science/article/pii/ S0022369708005374

320 [5] L. Liu, A. Corma, Metal Catalysts for Heterogeneous Catalysis: From Single Atoms to Nanoclusters and Nanoparticles, Chemical Reviews 118 (10) (2018) 4981-5079. doi:10.1021/acs.chemrev.7b00776.

URL https://doi.org/10.1021/acs . chemrev.7b00776

n [6] C. Bansal, S. G. Praveen, J. T. T. Kumaran, A. Chatterjee, Inter-cluster 325 distance dependence of electrical conduction in nanocluster assembled films of silver: a new paradigm for design of nanostructures, Scientific reports 5 (2015) 7685. doi:10.1038/srep07685 URL https://pubmed.ncbi.nlm.nih.gov/25566690https://www.ncbi. nlm.nih.gov/pmc/articles/PMC4286756/

330 ـ [7] F. Jiménez-Villacorta, C. Munuera, C. Ocal, C. Prieto, Study of nanocon1. ductive and magnetic properties of nanostructured iron films prepared by sputtering at very low temperatures, Journal of Nanoparticle Research 
12 (4) (2010) 1117-1127. doi:10.1007/s11051-009-9640-z.

URL https://doi.org/10.1007/s11051-009-9640-z

335 [8] M. Novotny, J. Bulir, J. Lancok, P. Pokorny, M. Bodnar, In-situ monitoring of the growth of nanostructured aluminum thin film, Journal of Nanophotonics 5 (1) (2011) 1-11. doi:10.1117/1.3543816.

URL https://doi.org/10.1117/1.3543816

[9] N. Abdellaoui, A. Pereira, M. Novotny, J. Bulir, P. Fitl, J. Lancok, B. Moine, A. Pillonnet, In situ monitoring of electrical resistance during a deposition of $\mathrm{Ag}$ and $\mathrm{Al}$ thin films by pulsed laser deposition: Compara-

a tive study, Applied Surface Science 418 (2017) 517-521. doi:10.1016/j. apsusc.2016.11.234

URL http://dx.doi.org/10.1016/j.apsusc.2016.11.234

345 [10] O. Kylián, J. Prokeš, O. Polonskyi, J. Čechvala, J. Kousal, J. Pešička, J. Hanuš, H. Biederman, Deposition and characterization of Pt nanocluster films by means of gas aggregation cluster source, Thin Solid Films 571 (P1) (2014) 13-17. doi:10.1016/j.tsf.2014.09.064.

[11] N. Maréchal, E. Quesnel, Y. Pauleau, Silver thin films deposited by magnetron sputtering, Thin Solid Films 241 (1) (1994) 34-38. doi:https://doi.org/10.1016/0040-6090(94)90391-3. URL http://www.sciencedirect.com/science/article/pii/ 0040609094903913

[12] K. Y. Chan, B. S. Teo, Sputtering power and deposition pressure effects on the electrical and structural properties of copper thin films,

q Journal of Materials Science 40 (22) (2005) 5971-5981. doi:10.1007/ s10853-005-1362-8.

[13] U. B. Arnalds, J. S. Agustsson, A. S. Ingason, A. K. Eriksson, K. B. Gylfason, J. T. Gudmundsson, S. Olafsson, A magnetron sputtering system for the preparation of patterned thin films and in situ thin film electrical 
resistance measurements, Review of Scientific Instruments 78 (10) (2007) 1-6. doi:10.1063/1.2793508.

[14] E. Barborini, G. Corbelli, G. Bertolini, P. Repetto, M. Leccardi, S. Vinati, P. Milani, The influence of nanoscale morphology on the resistivity of cluster-assembled nanostructured metallic thin films, New Journal of Physics 12 (7) (2010) 73001. doi:10.1088/1367-2630/12/7/073001 URL https://doi .org/10.1088\{\%\}2F1367-2630\{\%\}2F12\{\%\}2F7\{\%\}2F073001

[15] A. Sattar, S. Fostner, S. A. Brown, Quantized conductance and switching in percolating nanoparticle films., Phys. Rev. Lett. 111 (2013) 136808. doi: 10.1103/PhysRevLett.111.136808

[16] C. Minnai, A. Bellacicca, S. A. Brown, P. Milani, Facile fabrication of complex networks of memristive devices, Scientific Reports 7 (1) (2017) 1-8. doi:10.1038/s41598-017-08244-y

[17] M. Mirigliano, F. Borghi, A. Podestà, A. Antidormi, L. Colombo, P. Milani, Non-ohmic behavior and resistive switching of Au cluster-assembled films beyond the percolation threshold, Nanoscale Advances 1 (8) (2019) 31193130. doi:10.1039/c9na00256a.

[18] A. Vahl, N. Carstens, T. Strunskus, F. Faupel, A. Hassanien, Diffusive

n Memristive Switching on the Nanoscale, from Individual Nanoparticles towards Scalable Nanocomposite Devices, Scientific Reports 9 (1) (2019) 110. doi:10.1038/s41598-019-53720-2. URL http://dx.doi .org/10.1038/s41598-019-53720-2

[19] J. Schmelzer, S. A. Brown, A. Wurl, M. Hyslop, R. J. Blaikie, Finite-size effects in the conductivity of cluster assembled nanostructures, Phys. Rev. Lett. 88 (2002) 226802. doi:10.1103/PhysRevLett.88.226802. URL https://link.aps.org/doi/10.1103/PhysRevLett.88.226802

[20] V. Popok, Ion implantation of polymers: Formation of nanoparticulate materials, Reviews on Advanced Materials Science 30 (2012) 1-26. 
[21] M. C. Salvadori, M. Cattani, F. S. Teixeira, I. G. Brown, Conducting polymer formed by low energy gold ion implantation, Applied Physics Letters 93 (7) (2008) 73102. doi:10.1063/1.2973161.

URL https://doi.org/10.1063/1.2973161

[22] C. Binns (Ed.), Nanomagnetism: Fundamentals and Applications, Volume 6, 1st Edition, Elsevier, Amsterdam, Netherlands, 2014.

[23] S. Oyarzún, A. Tamion, F. Tournus, V. Dupuis, M. Hillenkamp, Size ef7. fects in the magnetic anisotropy of embedded cobalt nanoparticles: From n $\quad$ shape to surface, Scientific Reports 5 (August) (2015) 16-21. doi:10. 1038/srep14749.

URL http://dx.doi.org/10.1038/srep14749

[24] U. Heiz, U. Landman (Eds.), Nanocatalysis, Springer Berlin Heidelberg, 2007. doi:10.1007/978-3-540-32646-5. URL https://doi .org/10.1007\%2F978-3-540-32646-5

[25] P. Anger, P. Bharadwaj, L. Novotny, Enhancement and Quenching of Single-Molecule Fluorescence, Physical Review Letters 96 (11) (2006) 113002. doi:10.1103/PhysRevLett.96.113002.

URL https://link.aps.org/doi/10.1103/PhysRevLett.96.113002

[26] E. Cottancin, G. Celep, J. Lermé, M. Pellarin, J. R. Huntzinger, J. L. Vialle, M. Broyer, Optical Properties of Noble Metal Clusters as a Function of the Size: Comparison between Experiments and a Semi-Quantal Theory, Theoretical Chemistry Accounts 116 (4) (2006) 514-523. doi:10.1007/ s00214-006-0089-1.

URL https://doi.org/10.1007/s00214-006-0089-1

[27] A. R. Guerrero, R. F. Aroca, Surface-Enhanced Fluorescence with Shell-Isolated Nanoparticles (SHINEF), Angewandte Chemie International

15 Edition 50 (3) (2011) 665-668. doi:10.1002/anie.201004806.

URL https://onlinelibrary.wiley.com/doi/abs/10.1002/anie. 201004806 
[28] J. Ferreira, F. S. Teixeira, A. R. Zanatta, M. C. Salvadori, R. Gor$10.1039 / \mathrm{C} 2 \mathrm{CP} 23287 \mathrm{~A}$

URL http://dx.doi.org/10.1039/C2CP23287A

[29] D. Keith Roper, K. R. Berry, A. G. Russell, P. A. Blake, D. Keith Roper,

[30] O. N. Oliveira, R. M. Iost, J. R. Siqueira, F. N. Crespilho, L. Caseli, Nano1 materials for Diagnosis: Challenges and Applications in Smart Devices Based on Molecular Recognition, ACS Applied Materials \& Interfaces 6 (17) (2014) 14745-14766. doi:10.1021/am5015056.

URL https://doi.org/10.1021/am5015056

[31] L. A. Mercante, V. P. Scagion, A. Pavinatto, R. C. Sanfelice, L. H. C.

a Mattoso, D. S. Correa, Electronic Tongue Based on Nanostructured Hy${ }_{435}$ brid Films of Gold Nanoparticles and Phthalocyanines for Milk Analysis, Journal of Nanomaterials 2015 (2015) 890637. doi:10.1155/2015/890637 URL https://doi.org/10.1155/2015/890637

[32] S. Choudhury, R. Mangal, A. Agrawal, L. A. Archer, A highly rea versible room-temperature lithium metal battery based on crosslinked 440 hairy nanoparticles, Nature Communications 6 (1) (2015) 10101. doi: $10.1038 /$ ncomms 10101

URL https://doi .org/10.1038/ncomms10101

[33] A. D. T. de Sá, V. T. Abrao Oiko, G. di Domenicantonio, V. Rodrigues, n. New experimental setup for metallic clusters production based on hollow ${ }_{445} \quad$ cylindrical magnetron sputtering, Journal of Vacuum Science \& Technology 
B, Nanotechnology and Microelectronics: Materials, Processing, Measurement, and Phenomena 32 (6) (2014) 061804. doi:10.1116/1.4900847.

URL http://avs.scitation.org/doi/10.1116/1.4900847

[34] H. Haberland, M. Karrais, M. Mall, Y. Thurner, Thin films from energetic cluster impact: A feasibility study, Journal of Vacuum Science \& Technology A: Vacuum, Surfaces, and Films 10 (5) (1992) 3266-3271. doi:10.1116/1.577853,

[35] M. Hillenkamp, G. Di Domenicantonio, O. Eugster, C. Félix, Instability of Ag nanoparticles in SiO2 at ambient conditions, Nanotechnology 18 (2007) 015702. doi:10.1088/0957-4484/18/1/015702. URL https : //doi .org/10.1088/0957-4484/18/1/015702

[36] R. C. Hensel, K. L. Rodrigues, V. D. L. Pimentel, A. Riul, V. Rodrigues, Automated self-assembly and electrical characterization of nanostructured films, MRS Communications 8 (2) (2018) 283-288. doi:10.1557/mrc. 2018.47 .

[37] M. W. Den Otter, Approximate expressions for the capacitance and electrostatic potential of interdigitated electrode, Sensors and Actuators, A: Physical 96 (2-3) (2002) 140-144. doi:10.1016/S0924-4247(01)00783-X

[38] R. Igreja, C. J. Dias, Analytical evaluation of the interdigital electrodes capacitance for a multi-layered structure, Sensors and Actuators A: Physical 112 (2) (2004) 291-301. doi:https: //doi.org/10.1016/j.sna.2004.01.040

1 URL http://www.sciencedirect.com/science/article/pii/ S0924424704000779

470 [39] P. Grassberger, Conductivity exponent and backbone dimension in 2-d percolation, Physica A: Statistical Mechanics and its Applications 262 (3)

口 (1999) 251-263. doi:https://doi.org/10.1016/S0378-4371(98) 00435-X. 
URL http://www.sciencedirect.com/science/article/pii/

475 S037843719800435X

[40] A. K. Ahmad, B. L. Evans, A resistance network representation of electrical conduction in Pt and Cu films, Surface Science 364 (1) (1996)

39-53. doi:https://doi.org/10.1016/0039-6028(96)00589-4.

URL http://www.sciencedirect.com/science/article/pii/ 